\title{
Improvement of Coverage and Mobility in LTE-A Femto- Cell based on Cognitive Radio Network
}

\author{
Mahmoud Nagieb \\ Area manager at Systel Telecom
}

\author{
Mona Shokair \\ Assistant Professor at Faculty of Electronic \\ Engineering Menoufia University, Egypt
}

\begin{abstract}
The big challenge at any cellular system is to keep providing Coverage and quality of service. Femto-cells are used to overcome the dead zones problem and an efficient way to improve coverage. By using Cognitive Radio, spectral efficiency can be provided too. In this paper, a Femto-cell integrated over LTE Advanced (LTE-A) macro-cellular system under the effect of the distance between the macro user and the Femto-cell (R) on Signal-interference noise ratio (SINR), Path-Loss (PL) and Throughput (THR) with changing in bandwidth and the modulation technique are introduced which is not clarified until now. Moreover, studying of how it is important for the macro user to make handover at the Femto-cell to keep in coverage area.
\end{abstract}

\section{INTRODUCTION}

LTE-A is the next step in the wireless network evolution, it will enable higher than $1 \mathrm{Gbps}$ downlink bandwidth in addition to the existing LTE services. The 3rd Generation Partnership Project (3GPP) developed the following capabilities for LTE-Advanced, Wider bandwidth support Carrier aggregation up to $40 \mathrm{MHz}$ total band, and later potentially up to $100 \mathrm{MHz}$, MIMO evolution up to $8 \times 8$ in downlink and $4 \times 4$ in uplink Relay nodes for providing simple transmission solution, Heterogeneous networks for optimized interworking between cell layers including macro, micro, pico and Femto-cells [1].

Femto-cells or short-range antennas are introduced lately for indoor coverage extension, as it offers low power and low cost due to reducing cell sizes and the coverage distance per cell, Femto-cells can be used to overcome the dead zones to enhance the coverage. It can cooperate with a macrocell using the same band or different one [2].

Handover $(\mathrm{HO})$ is one of the key procedures for ensuring that the users move freely through the network while still being connected and being offered quality of services. HO can be done for many reasons as performance requirements (e.g. Bandwidth), cost, worse signal quality or loss sign and power consumption. HO can be classified to Horizontal and Vertical $\mathrm{HO}$ as shown in Figure 1.

Horizontal handover is made when a mobile terminal changes its point of connection within the same type of network (e.g. from a cell to another in GSM).

On the other hand, Vertical handover is considered when a mobile terminal changes its point of connection in a different type of network (e.g. from an access point in WiFi to a cell in UMTS) [3].

Cognitive Radio (CR) is used to solve the problems of spectrum inefficiency which CR provides the capability to use or share the spectrum in an opportunistic manner.

Dynamic spectrum access techniques allow the cognitive radio to operate in the best available channel, $\mathrm{CR}$ enables the usages of temporally unused spectrum which is referred to as spectrum hole or white space which is described in detail in [4 ], The main functions for CR are introduced in [4],[5].

In this paper, studying the coverage and mobility in LTE-A Femto-cell based on Cognitive Radio networks will be made. This study depends on investigating the effect of distance between macro user and Femto-cell on SINR, PL and THR under changing the bandwidth and different modulation techniques.

The rest of the paper is organized as follows, In Section 2, The system model will be presented, its specifications and the mathematical analysis used in it. In Section 3, the simulation results will be done. Finally, conclusions will be made in Section 4.

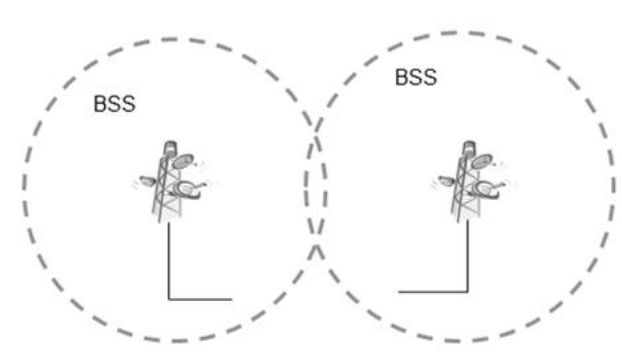

(a)

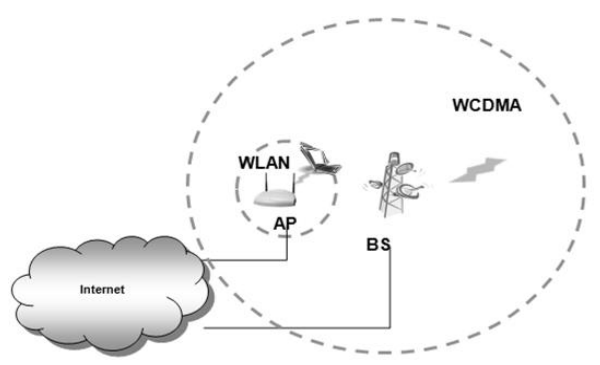

(b)

Fig 1: Horizontal and Vertical Handover. 


\section{SYSTEM MODEL}

The model simulates a Femto-cell integrated over LTE-A macrocellular system [6] as shown in Figure 2. The effect of the distance between the macro user and the Femto-cell on Signal-interference noise ratio (SINR), Path- loss (PL) and Throughput (THR) with changing the band-width and the modulation technique will be presented. In our simulation, the macro-user is considered as the secondary user and the Femto-user as the primary one, simulation parameters are illustrated at Table I.

\subsection{Path Loss Model}

Path-loss models describe the signal attenuation between a transmit and a receive antenna as a function of the propagation distance and other parameters like terrain contours, environment. Path-loss between a macro base station and user equipment (UE) [6] is calculated as follows:

$$
\mathrm{PL}(\mathrm{db})=15.3+37.6 \log 10 \mathrm{r}
$$

Where $r$ is the distance between the transmitter and the receiver in meters. The path loss between a femto base station and a UE is defined by the following Equation:

$\mathrm{PL}(\mathrm{db})=38.46+37.6 \log 10 \mathrm{r}+0.7 \mathrm{~d} 2$ D.indoor + $18.3 n((n+2) /(n+1)-0.46)+q^{*}$ Liw

Where $\mathrm{n}$ is the number of penetrated floor, $\mathrm{q}$ is the number of walls separating apartments between the femto base station and the UE, and Liw is the penetration loss of the wall separating apartments. Also, the term $0.7 \mathrm{~d} 2 \mathrm{D}$,indoor takes account of penetration loss due to walls inside an apartment and is expressed in $\mathrm{m}$.

\subsection{SINR Estimation}

SINR is Signal to Interference plus Noise Ratio commonly used in wireless communication as a way to measure the quality of wireless connections, The estimation of the received SINR of a macro user $m$ on subcarrier $k$, when the macro user is interfered from neighboring macrocells and all the adjacent Femto-cells, in our analysis is expressed by,

$$
\operatorname{SINR}_{\ldots}{ }_{m, k}=\frac{P_{M, k} G_{m, M, k}}{N_{o} \Delta . f+\sum_{M^{\prime}} P_{M^{\prime}, k} G_{m, M^{\prime}, k}+\sum_{F} P_{F, k} G_{m, F, k}}
$$

where $\mathrm{PM} ; \mathrm{k}$ and $\mathrm{PM}^{\prime} ; \mathrm{k}$ is transmit power of serving macrocell $\mathrm{M}$ and neighboring macrocell $\mathrm{M}^{\prime}$ on subcarrier $\mathrm{k}$, respectively. $\mathrm{Gm} ; \mathrm{M} ; \mathrm{k}$ is channel gain between macro user $\mathrm{m}$ and serving macrocell $\mathrm{M}$ on subcarrier $\mathrm{k}$. Channel gain from neighboring macrocells are denoted by $\mathrm{Gm} ; \mathrm{M}^{\prime} ; \mathrm{k}$. Similarly, $\mathrm{PF} ; \mathrm{k}$ is transmit power of neighboring femtocell $\mathrm{F}$ on subcarrier $\mathrm{k}$. Gm; $\mathrm{F} ; \mathrm{k}$ is channel gain between macro user $\mathrm{m}$ and neighboring femtocell $\mathrm{F}$ on subcarrier $\mathrm{k}$. N0 is white noise power spectral density, and $\Delta f$ is subcarrier spacing.
In case of a femto user $\mathrm{f}$ on subcarrier $\mathrm{k}$ interfered by all macrocells and adjacent femto-cells, the received SINR can be similarly given by,

$$
\operatorname{SINR}_{\boldsymbol{*}_{f, k}}=\frac{P_{F, k} G_{f, F, k}}{N_{o} \Delta \cdot f+\sum_{M} P_{M, k} G_{m, M, k}+\sum_{F} P_{F^{\prime}, k} G_{m, F^{\prime}, k}}
$$

The channel gain $G$ is affected by path-loss, it can be expressed as [1]:

$$
\mathrm{G}=10-\mathrm{PL} / 10
$$

\begin{tabular}{|c|c|c|c|c|c|c|}
\hline Parameter & \multicolumn{6}{|c|}{ Value } \\
\hline Macrocell Radius (Rm) & \multicolumn{6}{|c|}{$250 \mathrm{~m}$} \\
\hline Femtocell Radius (Rf) & \multicolumn{6}{|c|}{$20 \mathrm{~m}$} \\
\hline Frequency & \multicolumn{6}{|c|}{$2 \mathrm{GHZ}$} \\
\hline Macro BS Power & \multicolumn{6}{|c|}{$46 \mathrm{dBm}$} \\
\hline Femto BS Power & \multicolumn{6}{|c|}{$20 \mathrm{dBm}$} \\
\hline Outdoor Walls Loos & \multicolumn{6}{|c|}{$20 \mathrm{~dB}$} \\
\hline Indoor Walls Loss & \multicolumn{6}{|c|}{$5 \mathrm{~dB}$} \\
\hline Modulation Scheme & \multicolumn{2}{|c|}{ 64QAM } & \multicolumn{2}{|c|}{ 16QAM } & \multicolumn{2}{|c|}{ QPSK } \\
\hline Bandwidth (MHZ) & 20 & 15 & 10 & 5 & 3 & 1.4 \\
\hline Subcarrier Spacing & \multicolumn{6}{|c|}{$15 \mathrm{KHZ}$} \\
\hline White noise Power density & \multicolumn{6}{|c|}{$-174 \mathrm{dBm} / \mathrm{Hz}$} \\
\hline Number of Macro-user & \multicolumn{6}{|c|}{1} \\
\hline Number of Femto-user & & & & & & \\
\hline
\end{tabular}

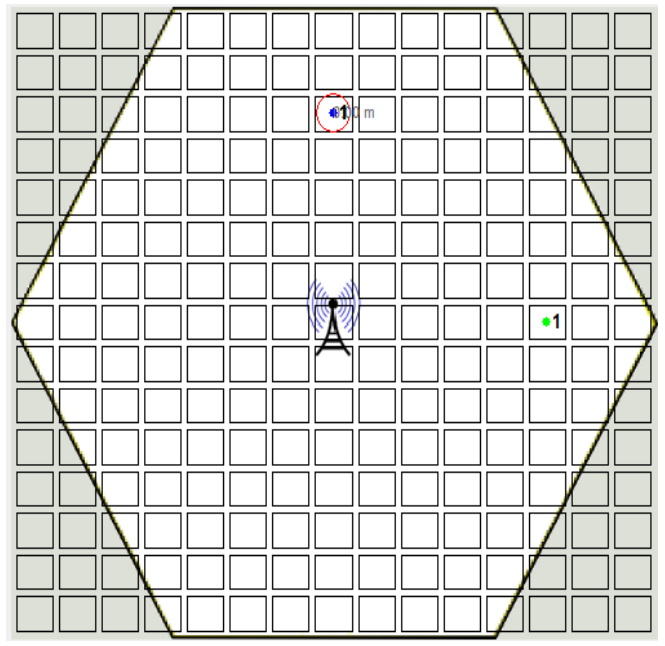

Fig 2: System Model.

Table I. System Parameters 


\subsection{Throughput Calculation}

The practical capacity of macro user $\mathrm{m}$ on subcarrier $\mathrm{k}$ can be given by the following equation:

$$
\mathrm{C}_{\mathrm{m}, \mathrm{k}}=\Delta \cdot f \log _{2}\left(1+\alpha \operatorname{SINR}_{\mathrm{m}, \mathrm{k}}\right)
$$

Where, $\alpha$ is a constant for target Bit Error Rate (BER), and defined by $\alpha=-1.5 / \ln$ (5BER). In this analysis BER is set to $10^{-6}$.

\section{SIMULATION RESULTS}

As mentioned before the effect of R on SINR, PL and THR with changing in bandwidth and modulation technique will be studied as shown in the following figures.

We found that as the user comes closer to the Femto-cell, it affects on him negatively, all Key Performance indicators (KPIs) totally degraded and become without coverage till the user handover on the Femto-cell, the KPIs clearly improved.

Figure 3 (a, b and c) shows a simple LTE-A Macro cell system using Band of $20 \mathrm{MHZ}$ and 64- QAM as a modulation technique. It shows the effect of the distance on SINR, THR and PL, respectively. From this Figures, we can conclude that as the user go away from the Macro BS, THR and SINR are decreasing with distance, PL fluctuating with distance as shown from points $1-5$ of X-Axis. SINR and THR are affected badly once the macro-user enters the Femto-cell coverage (point 6), after making handover the improvement in the KPIs are clear at point 7.

In Figure 4, another band of 5MHZ at QPSK is used. When we compare between Fig.3 and Fig.4, we can conclude that

We get nearly the same results from both assumptions except THR values are increased with using Band of $20 \mathrm{MHZ}$ and 64-QAM.
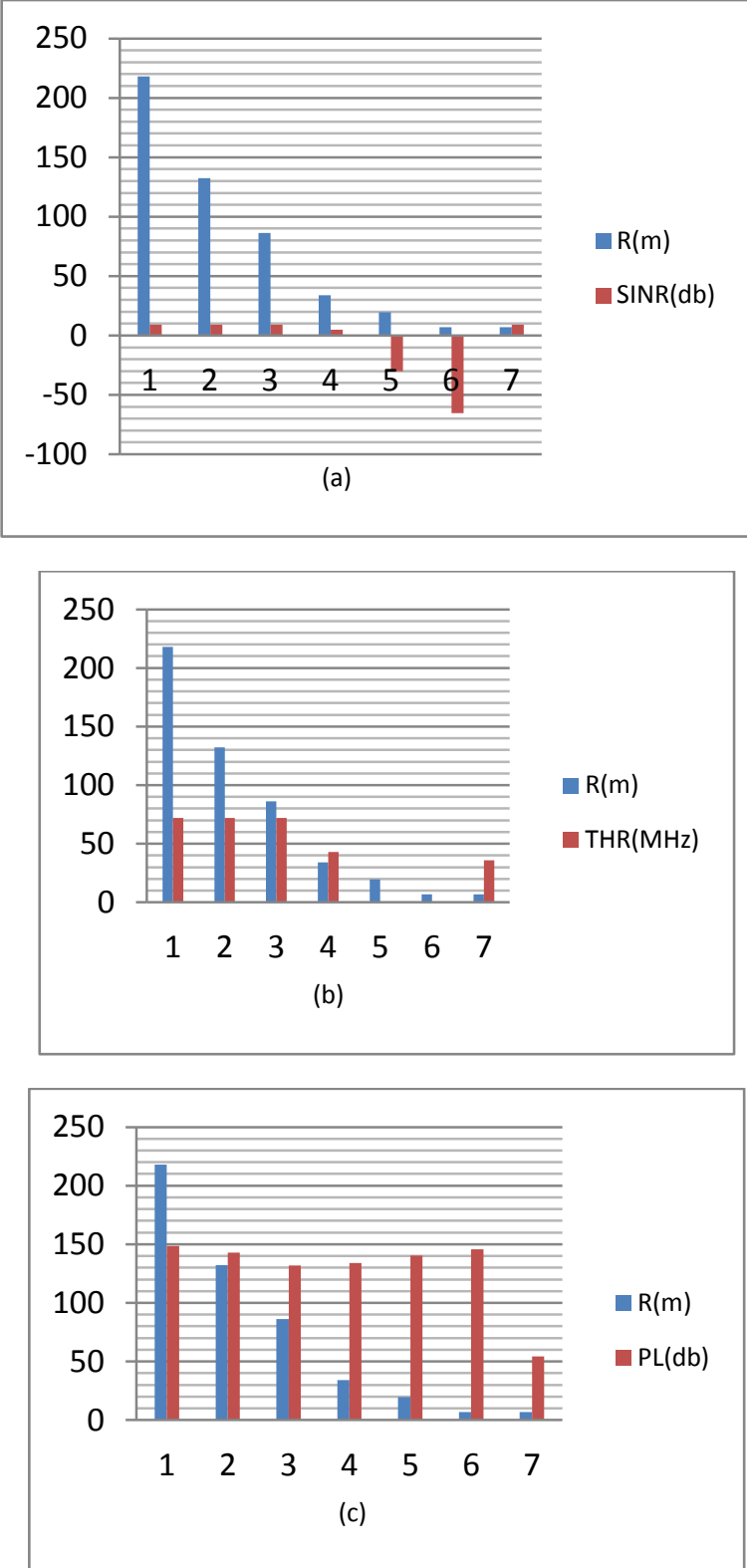

Fig 3: Relations between R and (SINR, THR and PL) at 20MHZ/64QAM
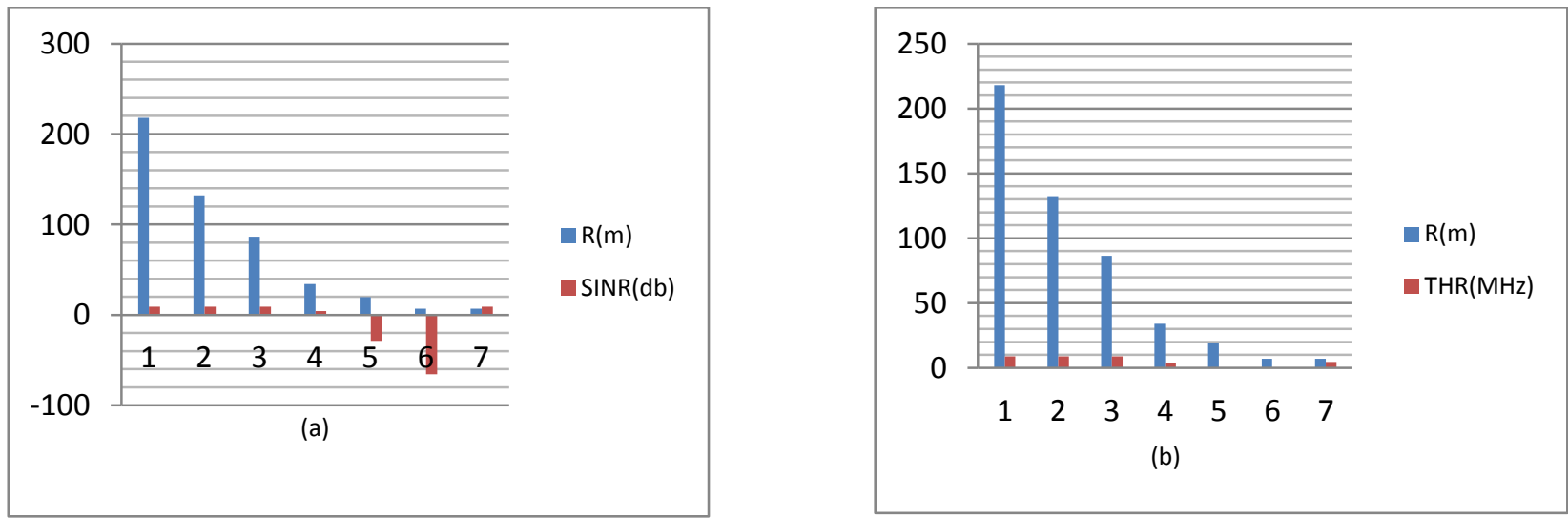


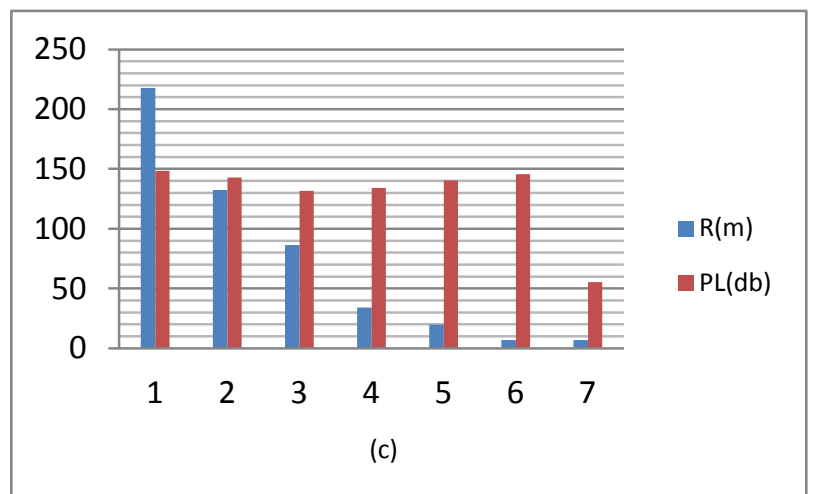

Fig 4: Relations between R and (SINR, THR and PL) at 5MHZ/QPSK

\section{CONCLUSIONS}

Femto-cells based on cognitive radio are an efficient way to improve coverage and overcome the dead zones. The objective of handover to achieve the mobility and keep covered without degradation at the quality of service and achieve user satisfaction. The effect of the distance between a macro-user and a Femto-cell (R) on SINR, THR and PL with changing the Bandwidth and the modulation technique was investigated. Our results show the effect of the distance on the macro user and its performance as its key performance indicator are varied according to the distance between him and the macro BS and the effect of the others interfering cells. We can see the effect of BW and modulation technique order on Throughput. As we use more BW and high modulation technique, throughput increases too.

\section{REFERENCES}

[1] A.Z.Yonis and M.F.L.Abdullah, "Peak-Throughput of LTE-Release 10 for Up/Down Link Physical Layer, International Journal of Information \& Network Security (IJINS), Vol.1, No.2, pp. 88-96, June 2012.

[2] Chowdhury.M.Z. Minh Trung Bui and Yeong Min Jang, "Neighbor Cell List Optimization for Femtocell-to-
Femtocell Handover in Dense Femtocellular Networks", Ubiquitous and Future Networks (ICUFN), pp. 241-245, June 2011.

[3] Y.Zhang, "Spectrum Handoff in Cognitive Radio Networks: Opportunistic and Negotiated Situations", Communications, 2009. ICC '09. IEEE International Conference, pp. 1-6, June 2009.

[4] I.F.Ak.yildiz, W-Y.Lee, M.C.Vuran and S.Mohanty, "NeXt Generation/Dynamic Spectrum Access/Cognitive Radio Wireless Networks: A Survey", Computer Networks: The International Journal of Computer and Telecommunications Networking, Vol.50, Issue 13, pp. 2127-2159, September 2006.

[5] N.Alyaoui, A.Kachouri and M. Samet, "The Fourth Generation 3GPP LTE Identification for Cognitive Radio", Microelectronics (ICM), 2011 International Conference, pp. 19-22, December 2011.

[6] C.Bouras, V.Kokkino, K.Kontodimas and A.Papazois, "A Simulation Framework for LTE-A Systems with Femtocell Overlays", MSWiM Modeling, Analysis and Simulation of Wireless and Mobile Systems, pp. 85-90, 2012. 